\title{
Burden of Chronic Sleep Maintenance Insomnia Characterized by Nighttime Awakenings
}

\author{
Susan C. Bolge, Ph.D., Vijay N. Joish, Ph.D., ${ }^{2}$ Rajesh Balkrishnan, Ph.D., ${ }^{3}$ \\ Hema Kannan, M.P.H., and Christopher L. Drake, Ph.D. ${ }^{4}$
}

\begin{abstract}
The objective of this study was to evaluate the economic and humanistic burden of chronic sleep maintenance insomnia characterized by nighttime awakenings (CINA). A database analysis of National Health and Wellness Survey, an annual cross-sectional study of health status and outcomes of US adults, was performed. CINA was defined as experiencing nighttime awakenings at least twice per week for more than 1 month that have moderate to severe impact on daily life and not experiencing difficulty falling asleep. No insomnia was defined as not selfreporting insomnia, sleep difficulties, or sleep symptoms. Outcomes included resource utilization in the past 6 months, the Work Productivity and Activity Impairment questionnaire, and the SF-8. Linear regression models were developed to assess the independent effects of CINA on outcomes while controlling for demographics and comorbidity.

In all, 1523 respondents met the criteria for CINA and 24,106 met the criteria for no insomnia. Controlling for demographics and comorbidity, CINA sufferers had greater resource utilization $(0.1[P<0.001]$ more emergency room visits, $0.2[P=0.001]$ more days hospitalized, and $2.5[P<0.001]$ more provider visits $), 22.4 \%(P<0.001)$ greater activity impairment, and SF-8 physical and mental summary scores that were $6.2(P<0.001)$ and 6.8 $(P<0.001)$ points lower than those with no insomnia, respectively. Among those employed full time, CINA sufferers had greater work productivity impairment ( $4.0 \%$ due to absenteeism, $17.6 \%$ due to presenteeism, and $15.6 \%$ greater overall productivity impairment) than those with no insomnia $(P<0.001$ for all).

CINA in relative isolation was associated with a significant negative impact on health care utilization and its associated costs, health-related quality of life, and work productivity. (Population Health Management 2010;13:15-20)
\end{abstract}

\section{Introduction}

$\mathbf{I}_{\mathrm{r}}^{\mathrm{s}}$ NSOMNIA IS DEFINED AS A SYNDROME characterized by problems falling asleep, staying asleep, waking up too early, or not feeling rested even after ample time in bed; it is associated with impairment in daytime functioning by the International Classification of Sleep Disorders and the Diagnostic and Statistical Manual of Mental Disorders. ${ }^{1,2}$ Currently, chronic insomnia affects between $9 \%$ and $12 \%$ of the general population. ${ }^{3}$ Based on data from the National Comorbidity Survey Replication, during a 12-month period $16.4 \%$ of adults experienced difficulty initiating sleep, $19.9 \%$ experienced difficulty maintaining sleep, $16.7 \%$ experienced early morning awakening, and $25.0 \%$ experienced nonrestorative sleep. ${ }^{4}$ Approximately $20 \%$ of patients who visited primary care settings reported experiencing "severe and persistent insomnia." ${ }^{15}$ The literature indicates that chronic insomnia is more prevalent than heart disease, cancer, diabetes, and gastrointestinal problems. ${ }^{6}$

The negative effects of insomnia have been well documented. Insomnia has been associated with various adverse effects including severe fatigue, irritability, mood disorders, and memory troubles. ${ }^{2,7,8}$ Insomnia sufferers complain of impaired daily functioning, which in turn has serious implications for overall quality of life and work productivity. ${ }^{7}$ One study found that both mental health status and emotional dimensions of quality of life were lower among severe and mild insomniacs compared to those with no insomnia. ${ }^{7}$ In a study of managed care enrollees by Hatoum et al, ${ }^{9}$ those with insomnia reported significantly lower health-related quality

\footnotetext{
${ }^{1}$ Consumer Health Sciences, Princeton, New Jersey (at time of writing).

${ }^{2}$ Sanofi-Aventis, Bridgewater, New Jersey (at time of writing).

${ }^{3}$ Schools of Pharmacy and Public Health, The University of Michigan Ann Arbor, Michigan.

${ }^{4}$ Henry Ford Hospital Sleep Center, and Psychiatry and Behavioral Neurosciences, Wayne State College of Medicine, Detroit, Michigan.
} 
of life (HRQOL) scores when compared to those with no insomnia.

The overall cost burden associated with insomnia has been quantified in some previous literature. Ozminkowski et al ${ }^{10}$ reported that average direct and indirect costs were significantly higher (\$1253 more) for insomniacs compared to those without insomnia. Insomnia has also been noted to have an effect on indirect costs, specifically work productivity and absence. Significantly higher rates of insomnia-related absenteeism have been documented in several different studies. ${ }^{10,11}$ A study by Leger et $\mathrm{al}^{12}$ noted that insomniacs missed work twice as often as good sleepers (odds ratio $=1.93, P<0.001$ ).

The direct costs associated with insomnia have been more extensively quantified than the indirect costs. Individuals who experience insomnia have been reported to have higher resource utilization, including emergency room visits, hospitalizations, and physician visits, when compared to those who do not experience insomnia. 9,13 The annual direct cost of insomnia is estimated to be approximately $\$ 14$ billion in the United States. ${ }^{14}$

Though the prevalence of insomnia and the burden of illness associated with insomnia have been addressed in the literature, less focus has been given to determining the impact of specific symptoms of insomnia. As noted earlier, 2 symptoms of insomnia are difficulty staying asleep or early morning awakening, hereafter collectively referred to as chronic sleep maintenance insomnia characterized by nighttime awakenings (CINA). In light of recent pharmacological advances in the treatment of insomnia that target specific symptoms of insomnia, there is a need to understand the burden of illness associated in a symptom-specific manner. The objective of this study was to evaluate the economic and humanistic burden of CINA without difficulty falling asleep using a nationwide sample.

\section{Methods}

\section{Study design}

Data were obtained from the 2006 United States National Health and Wellness Survey (NHWS) (Consumer Health Sciences, Princeton, NJ), an annual cross-sectional study of the disease status, health care attitudes, behaviors, and outcomes of the adult population aged 18 and older. The NHWS sample was identified through a Web-based consumer panel and was stratified by sex, age, and race/ethnicity so as to reflect the demographic composition of the total US adult population. Data were collected from respondents through a self-administered, Internet-based questionnaire. The NHWS study protocol and questionnaire were reviewed and approved by the Essex Institutional Review Board (Lebanon, NJ). Informed consent was obtained prior to entering the survey.

\section{Study measures}

Chronic sleep maintenance insomnia characterized by nighttime awakenings versus no insomnia. All NHWS respondents were asked to report any experience with symptoms of insomnia, consistent with the National Institutes of Health definition of comorbid insomnia and the Diagnostic and Statistical Manual of Mental Disorders, Fourth Edition, Text Revision criteria for nonprimary insomnia, ${ }^{2,15}$ and the impact these symptoms have on their personal and professional lives. Specifically, respondents reported experiencing any of the following more than 2 times a week during the past year: difficulty falling asleep; difficulty staying asleep-either waking several times during the night or waking and not being able to get back to sleep (nighttime awakenings); waking up too early before the alarm clock (nighttime awakenings). In addition to symptoms, respondents also reported whether they had experienced insomnia or sleep difficulties in the past 12 months and, if so, quantified the duration and frequency of sleep difficulties.

Respondents were categorized as experiencing CINA if they met the following criteria: experienced 1 or both nighttime awakenings symptoms, did not experience difficulty falling asleep, experienced sleep difficulties at least twice per week for a period of more than 1 month, and symptoms had a moderate to severe impact on personal and professional life. Respondents were categorized as no insomnia (control group) if they met the following criteria: did not self-report insomnia or sleep difficulties in the past 12 months and did not experience difficulty falling asleep or nighttime awakenings as described earlier.

Comorbid conditions. A count of physical health conditions was computed for each respondent. The following conditions were included in the count: angina, arrhythmia, arthritis, asthma, atrial fibrillation, chronic obstructive pulmonary disorder, congestive heart failure, deep vein thrombosis, diabetes, epilepsy, high blood pressure, high cholesterol, inflammatory bowel disease, irritable bowel syndrome, migraine, nasal allergies, osteoporosis, overactive bladder, peripheral arterial disease, peripheral vascular disease, psoriasis, thyroid condition. Respondents were categorized as experiencing a psychiatric condition if they reported experiencing any of the following: anxiety, bipolar disorder, depression, generalized anxiety disorder, obsessive-compulsive disorder, panic disorder, phobias, post-traumatic stress disorder, or social anxiety disorder.

Resource utilization (direct costs). Resource utilization was assessed for the prior 6 months and included number of emergency room visits, number of days hospitalized, and number of visits to all medical providers.

Lost work productivity and activity impairment (indirect costs). The Work Productivity and Activity Impairment (WPAI) questionnaire was used to assess lost work productivity and activity impairment. The WPAI is appropriate for use across occupations and disease areas and has been used to assess health-related work productivity more frequently than any other metric. WPAI measures are expressed as impairment percentages with higher values indicating a greater proportion of impairment. Specific impairment metrics for work productivity include absenteeism (work time missed due to health problems), presenteeism (impairment at work), and overall work productivity loss (combination of absenteeism and presenteeism). Lost work productivity measures were assessed only for respondents who work full time. Activity impairment is a single measure of impairment due to health. ${ }^{16,17}$

Health-related quality of life. HRQOL was assessed using the Medical Outcomes Study Short-Form Health Sur- 
vey (SF-8). The SF-8 is a validated metric based on the SF-36, which has been widely used to compare the burden of different diseases, to differentiate health benefits or treatments, and to screen patients across a wide variety of therapeutic areas. The 8 questions included in the SF- 8 are scores on the same norm-based metric as the SF-36 scales (ie, physical functioning, role limitations due to physical health problems, bodily pain, general health, vitality, social functioning, role limitations due to emotional problems, mental health) and summary scores. The physical and mental summary scores are normed for the US adult population with a mean of 50 and a standard deviation of $\pm 10 .^{18,19}$

\section{Statistical analysis}

A comparison of demographic profiles, comorbid conditions, and outcomes of CINA sufferers and noninsomnia sufferers was first examined at the bivariate level. Chi-square was used to test for significant differences in categorical variables. Analysis of variance was used to test for significant differences in continuous variables.

Linear regression models were developed to assess the independent effects of CINA on outcomes. Separate models were developed for each of the following dependent variables: SF-8 physical component summary score, SF-8 mental component summary score, WPAI absenteeism, WPAI presenteeism, WPAI overall work productivity loss, WPAI activity impairment, number of emergency room visits, number of days hospitalized, and number of visits to traditional medical providers. Potential confounders controlled for in the models included sex, age, race, number of comorbid conditions, and experiencing a psychiatric condition.

\section{Results}

\section{Sample characteristics}

Of the 62,833 respondents to the 2006 US NHWS, 1523 $(2 \%)$ met the criteria for experiencing CINA, and 24,106 (38\%) met the criteria for no insomnia. Among respondents who were employed full time, 496 met the criteria for experiencing CINA and 10,346 met the criteria for no insomnia.

CINA sufferers were significantly more likely to be female and older than those with no insomnia. CINA sufferers were less likely to be nonwhite; specifically, CINA sufferers were less likely than those with no insomnia to be African American, Asian, or Hispanic and more likely to be American Indian or of mixed race. CINA sufferers also experienced twice as many physical comorbid conditions, were more likely to experience each physical comorbid condition individually, and were nearly 4 times as likely as those with no insomnia to experience a psychiatric condition, the most common of which were depression and generalized anxiety disorder (Table 1).

\section{Direct costs}

On average, CINA sufferers had 0.4 emergency room visits, 0.8 days hospitalized, and 8.0 visits to traditional medical providers in the prior 6 months (Table 2). This resource utilization was significantly greater than for those with no insomnia $(P<0.001$ for all). Controlling for demographic and comorbidity differences, CINA sufferers had 0.1 more emergency room visits, 0.2 more days hospitalized, and 2.5 more visits to traditional providers in a 6-month period than those with no insomnia (Table 3).

\section{Indirect costs}

Though CINA sufferers were significantly less likely to work full time than those with no insomnia (33\% vs. $43 \%$, $P<0.001)$, CINA sufferers who worked full time experienced a substantial magnitude of work impairment, specifically experiencing $8.5 \%$ productivity impairment due to absenteeism, $34.4 \%$ productivity impairment while working (presenteeism), and $30.0 \%$ overall work productivity impairment. Based on unadjusted, bivariate analysis, CINA sufferers experienced a significantly greater loss of work productivity that was more than 3 times higher than for those with no insomnia (Table 2). Adjusting for demographics and comorbidity, CINA sufferers had $4.0 \%$ greater productivity impairment due to absenteeism, $17.6 \%$ greater productivity impairment while working (presenteeism), and 15.6\% greater overall work productivity impairment than those with no insomnia (Table 3).

CINA sufferers also experienced a significantly greater burden of activity impairment, reporting an average of $52 \%$ activity impairment, which was more than 3 times higher than that reported by those with no insomnia (Table 2). Adjusting for demographics and comorbidity, CINA sufferers had $22.4 \%$ greater activity impairment than those with no insomnia (Table 3).

\section{Health-related quality of life}

CINA sufferers had average SF-8 physical component summary scores of 40.0 and average SF- 8 mental component summary scores of 40.9 , which were not only significantly poorer than those with no insomnia but notably lower than the normative means of 50 for the US adult population (Table 1). Adjusting for demographics and comorbidity, CINA sufferers had SF-8 physical component summary scores that were 6.2 points lower and SF- 8 mental component summary scores that were 6.8 points lower than those with no insomnia (Table 3).

\section{Discussion}

Insomnia is a widely prevalent condition, with more than $10 \%$ of the adult population experiencing chronic insomnia. ${ }^{20}$ The impact of insomnia on direct and indirect costs has been documented elsewhere. ${ }^{10,13}$ The majority of research concerning this condition has focused on the diagnosis rather than specific symptoms. This study was conducted to quantify the impact of CINA without difficulty falling asleep on direct and indirect costs and HRQOL outcomes.

Based on data from the National Comorbidity Survey Replication, there are 37 million adults in the United States who experience insomnia characterized by nighttime awakenings. ${ }^{4}$ The criteria for CINA in this study were considerably more conservative, including only adults who experienced nighttime awakenings at least 3 times per week for more than 1 month that caused moderate to severe impact on daily life and who did not experience any symptoms associated with sleep induction. Projecting the NHWS data to the US adult population, there were 5 million adults who meet this strict criteria for CINA. 
Table 1. Sample Characteristics of Respondents Experiencing Chronic Sleep Maintenance Insomnia Characterized by NightTime AwaKenings (CINA) versus No Insomnia

\begin{tabular}{|c|c|c|c|}
\hline & \multirow{2}{*}{$\frac{\text { CINA }}{(\mathrm{n}=1523)}$} & \multirow{2}{*}{$\frac{\text { No Insomnia }}{(\mathrm{n}=24,106)}$} & \multirow[b]{2}{*}{ P value } \\
\hline & & & \\
\hline$\%$ Female & $59 \%$ & $45 \%$ & $<0.001$ \\
\hline Age-Mean (SD) & $50.6(13.9)$ & $48.9(16.8)$ & $<0.001$ \\
\hline $\begin{array}{l}\text { \% Nonwhite } \\
\text { African American } \\
\text { American Indian } \\
\text { Asian } \\
\text { Hispanic } \\
\text { Mixed } \\
\text { Other }\end{array}$ & $\begin{array}{l}18 \% \\
7.8 \% \\
2.2 \% \\
1.0 \% \\
4.5 \% \\
2.2 \% \\
0.5 \%\end{array}$ & $\begin{array}{l}23 \% \\
8.7 \% \\
0.9 \% \\
5.4 \% \\
6.0 \% \\
1.3 \% \\
0.7 \%\end{array}$ & $<0.001$ \\
\hline Count of physical comorbid conditions-Mean (SD) & $3.4(2.2)$ & $1.6(1.6)$ & $<0.001$ \\
\hline \multicolumn{4}{|l|}{ Physical comorbid condition } \\
\hline Ángina & $8.9 \%$ & $3.2 \%$ & $<0.001$ \\
\hline Arrhythmia & $7.3 \%$ & $2.6 \%$ & $<0.001$ \\
\hline Arthritis & $45.3 \%$ & $19.3 \%$ & $<0.001$ \\
\hline Asthma & $13.9 \%$ & $6.3 \%$ & $<0.001$ \\
\hline Atrial fibrillation & $3.2 \%$ & $1.6 \%$ & $<0.001$ \\
\hline Chronic obstructive pulmonary disorder & $6.7 \%$ & $2.1 \%$ & $<0.001$ \\
\hline Congestive heart failure & $5.4 \%$ & $1.3 \%$ & $<0.001$ \\
\hline Deep vein thrombosis & $2.1 \%$ & $0.8 \%$ & $<0.001$ \\
\hline Diabetes & $19.7 \%$ & $10.3 \%$ & $<0.001$ \\
\hline Epilepsy & $0.7 \%$ & $0.5 \%$ & 0.289 \\
\hline High blood pressure & $45.6 \%$ & $29.4 \%$ & $<0.001$ \\
\hline High cholesterol & $44.5 \%$ & $28.7 \%$ & $<0.001$ \\
\hline Inflammatory bowel disease & $1.5 \%$ & $0.4 \%$ & $<0.001$ \\
\hline Irritable bowel syndrome & $15.5 \%$ & $3.7 \%$ & $<0.001$ \\
\hline Migraine & $27.8 \%$ & $10.1 \%$ & $<0.001$ \\
\hline Nasal allergies & $38.5 \%$ & $19.2 \%$ & $<0.001$ \\
\hline Osteoporosis & $7.9 \%$ & $3.3 \%$ & $<0.001$ \\
\hline Overactive bladder & $17.6 \%$ & $3.7 \%$ & $<0.001$ \\
\hline Peripheral arterial disease & $3.0 \%$ & $0.9 \%$ & $<0.001$ \\
\hline Peripheral vascular disease & $1.2 \%$ & $0.4 \%$ & $<0.001$ \\
\hline Psoriasis & $6.3 \%$ & $2.7 \%$ & $<0.001$ \\
\hline Thyroid condition & $15.6 \%$ & $7.6 \%$ & $<0.001$ \\
\hline$\%$ With psychiatric condition & $62 \%$ & $16 \%$ & $<0.001$ \\
\hline Bipolar disorder & $5 \%$ & $1 \%$ & $<0.001$ \\
\hline Depression & $50 \%$ & $10 \%$ & $<0.001$ \\
\hline Generalized anxiety disorder & $23 \%$ & $4 \%$ & $<0.001$ \\
\hline Obsessive-compulsive disorder & $5 \%$ & $1 \%$ & $<0.001$ \\
\hline Panic disorder & $12 \%$ & $2 \%$ & $<0.001$ \\
\hline Phobias & $4 \%$ & $1 \%$ & $<0.001$ \\
\hline Post-traumatic stress disorder & $9 \%$ & $1 \%$ & $<0.001$ \\
\hline Social anxiety disorder & $14 \%$ & $2 \%$ & $<0.001$ \\
\hline
\end{tabular}

\footnotetext{
${ }^{\$} P$-values are computed using analysis of variance for continuous variables and chi-square for categorical variables.
}

CINA was associated with increased direct health care costs. Based on results of 2003-2004 Medical Expenditure Panel Survey (MEPS) and the 2005 National Hospital Discharge Survey (NHDS), the 0.1 greater emergency room visit associated with CINA equated to $\$ 56(0.1 *$ MEPS mean cost per emergency room visit $\left.[\$ 560]^{21}\right)$, the 0.2 more days hospitalized equated to $\$ 162(0.2 *$ MEPS cost per hospital visit $[\$ 3879]^{22} /$ NHDS average days per visit $\left.[4.8]^{23}\right)$, and the 2.5 additional provider visits equated to $\$ 388$ (2.5* MEPS cost per provider visit $\left.[\$ 155]^{24}\right)$. Overall, CINA was associated with an estimated increase in health care expenditures of \$606 in a 6 -month period or $\$ 1212$ per year per sufferer. Projecting this cost to the 5 million CINA sufferers yields an annual cost of $\$ 6.06$ billion in direct costs.
Not only was CINA associated with greater direct health care costs, it also was associated with an increase in indirect costs through loss of work productivity and increased impairment of daily activities. The work productivity loss experienced by CINA sufferers was more greatly affected by impairment while working (presenteeism) than actual lost work time (absenteeism). Overall, CINA sufferers had 16\% greater work productivity loss than those with no insomnia. This $16 \%$ equated to a loss of 6.4 hours of work during a 40-hour workweek. Assuming a work year of 50 weeks, CINA was associated with an additional 320 hours or 8 weeks of lost productivity per year per sufferer, compared to those with no insomnia. An even greater proportion (22\%) of activity impairment was associated with CINA. 
Table 2. Unadjusted Effects of Chronic Sleep Maintenance Insomnia Characterized by Nighttime Awakenings (CINA) on Economic and Humanistic Outcomes ${ }^{\star}$

\begin{tabular}{|c|c|c|c|}
\hline & CINA & No Insomnia & $\mathrm{P}$ value \\
\hline Direct Costs in Past 6 Months & $(n=1523)$ & $(\mathrm{n}=24,106)$ & \\
\hline Emergency room visits & $0.4(0.9)$ & $0.1(0.7)$ & $<0.001$ \\
\hline Days hospitalized & $0.8(4.2)$ & $0.3(2.4)$ & $<0.001$ \\
\hline Traditional medical provider visits & $8.0(11.9)$ & $3.3(4.6)$ & $<0.001$ \\
\hline Indirect Costs (WPAI) & $(n=496)$ & $(\mathrm{n}=10,346)$ & \\
\hline$\%$ Work full time & $33 \%$ & $43 \%$ & $<0.001$ \\
\hline Absenteeism $^{\dagger}$ & $8.5(21.2)$ & $2.8(12.3)$ & $<0.001$ \\
\hline Presenteeism $^{\dagger}$ & $34.4(25.4)$ & $10.4(19.2)$ & $<0.001$ \\
\hline Overall work productivity loss ${ }^{\dagger}$ & $30.0(30.8)$ & $9.0(20.3)$ & $<0.001$ \\
\hline & $(n=1523)$ & $(\mathrm{n}=24,106)$ & \\
\hline Activity impairment & $52.0(29.1)$ & $16.8(24.9)$ & $<0.001$ \\
\hline HRQOL (SF-8) & $(\mathrm{n}=1523)$ & $(\mathrm{n}=24,106)$ & \\
\hline Physical summary score & $40.0(11.0)$ & $50.4(8.5)$ & $<0.001$ \\
\hline Mental summary score & $40.9(11.1)$ & $52.8(7.6)$ & $<0.001$ \\
\hline
\end{tabular}

${ }^{\dagger}$ Among respondents who are employed full time.

Table shows mean (SD) values for each metric not specified by \%. $P$-values are computed using analysis of variance for continuous variables and chi-square for categorical variables.

HRQOL, health-related quality of life; SF-8, Short Form 8; WPAI, Work Productivity and Activity Impairment.

Study results also indicated that CINA had a strong negative effect on HRQOL. A 5 point change in the SF-36, and by extension the SF-8, has been cited as a clinically meaningful difference. ${ }^{19}$ After controlling for potential confounders, the difference in SF-8 physical and mental component summary scores between CINA sufferers and those with no insomnia was not only statistically different but had meaningful clinical implications.

This study had several limitations, mainly with respect to data collection. Subjects were enrolled from an Internet panel, potentially yielding a nonrepresentative sample of the general US population. The possibility that 1 segment of the general US population (the lowest socioeconomic groups, those residing in rural areas, or those who choose not to use the Internet) might have been excluded needs to be addressed. ${ }^{25}$ Use of the Internet for this study also may limit the diversity of the sample. However, there is evidence in the literature to indicate that a substantial portion of the US population does have Internet access. Approximately $70 \%$ of adults in the United States had Internet access in $2006 .{ }^{26}$

Second, results were based on self-reported measures. These self-reports were not verified against physician diagnoses or chart reviews. Self-report of comorbid conditions without verification against diagnosis criteria may result in overestimation, especially of psychiatric conditions. There was also the potential for recall bias. Because of the cross-sectional nature of this study, specific metrics such as HRQOL and work productivity could have differed had they been measured prospectively. Lastly, though we adjusted for demographics and comorbidity, there may have been other potential confounders that were not considered such as use of medications that may promote either sleep or wakefulness, or interactions of comorbidities with CINA. Future research should consider additional factors that may affect the burden of CINA.

Table 3: Summary of Adjusted ${ }^{\ddagger}$ Effects of Experiencing CiNA (1) versus No Insomnia (0) on Economic ANd Humanistic Outcomes $(\mathrm{N}=25,629)$

\begin{tabular}{|c|c|c|c|c|}
\hline & \multirow[b]{2}{*}{ beta } & \multirow[b]{2}{*}{ P value } & \multicolumn{2}{|c|}{$95 \% C I$} \\
\hline & & & Low & High \\
\hline \multicolumn{5}{|l|}{ Direct Costs in Past 6 Months } \\
\hline Emergency room visits & 0.094 & $<0.001$ & 0.056 & 0.132 \\
\hline Days hospitalized & 0.229 & 0.001 & 0.088 & 0.369 \\
\hline Traditional medical provider visits & 2.545 & $<0.001$ & 2.267 & 2.822 \\
\hline \multicolumn{5}{|l|}{ Indirect Costs (WPAI) } \\
\hline Absenteeism ${ }^{\dagger}$ & 3.950 & $<0.001$ & 2.721 & 5.180 \\
\hline Presenteeism ${ }^{\dagger}$ & 17.563 & $<0.001$ & 15.722 & 19.404 \\
\hline Overall work productivity loss ${ }^{\dagger}$ & 15.599 & $<0.001$ & 13.638 & 17.561 \\
\hline Activity impairment & 22.361 & $<0.001$ & 21.056 & 23.666 \\
\hline \multicolumn{5}{|l|}{ HRQOL (SF-8) } \\
\hline Physical summary score & -6.245 & $<0.001$ & -6.675 & -5.815 \\
\hline Mental summary score & -6.809 & $<0.001$ & -7.176 & -6.441 \\
\hline
\end{tabular}

\footnotetext{
${ }^{\dagger}$ Among respondents who are employed full time. Sample size of full-time employed is 10,840 .

Linear regression models adjust for sex, age, race, number of physical comorbid conditions, and experiencing a psychiatric condition. HRQOL, health-related quality of life; SF-8, Short Form 8; WPAI, Work Productivity and Activity Impairment.
} 
Although there is a significant burden of illness associated with insomnia, the present findings suggest that CINA in relative isolation is associated with a significant negative impact on quality of life, health care utilization, and work productivity. Further, CINA may negatively affect direct costs with respect to emergency room visits and hospitalizations as well as indirect costs. Additional research is needed to quantify the effects of CINA in specific populations as well as the burden of other insomnia symptoms and the effects of treatment.

\section{Disclosure Statement}

At the time of the research and manuscript development, Dr. Bolge and Ms. Kannan were employees of Consumer Health Sciences. Dr. Bolge is now an employee of Centocor Ortho Biotech Services, Horsham, PA. At the time of research and manuscript development, Dr. Joish was an employee of Sanofi-Aventis. He is now an employee of Bayer. Dr. Balkrishnan is a paid consultant for Sanofi-Aventis, Merck and Company, Novo Nordisk, Glaxo SmithKline, Bristol MyersSquibb, and Takeda Pharmaceuticals. In addition he receives grant support from Galderma. Dr. Drake conducts research for Cephalon and Takeda, has been paid as a speaker for Cephalon, and has consulted for Sanofi-Aventis and Takeda. The study used data from the National Health and Wellness Survey (NHWS), which is conducted by Consumer Health Sciences, Princeton, NJ. Sanofi-Aventis, Bridgewater, NJ, licensed access to NHWS and funded the analysis presented in this article.

\section{References}

1. American Sleep Disorders Association. International Classification of Sleep Disorders: Diagnostic and Coding Manual (ICSD). Rochester, MN: American Sleep Disorders Association; 1990.

2. American Psychiatric Association. Diagnostic and Statistical Manual of Mental Disorders (4 $4^{\text {th }}$ Edition) (DSM-IV). Washington DC: American Psychiatric Association; 1994.

3. Taylor DJ, Lichstein KL, Durrence HH, Reidel BW, Bush AJ. Epidemiology of insomnia, depression, and anxiety. Sleep 2005;28:1457-1464.

4. Roth T, Jaeger S, Jin R, Kalsekar A, Stang PE, Kessler RC. Sleep problems, comorbid mental disorders, and role functioning in the national comorbidity survey replication. Biol Psychiat 2006;60:1364-1371.

5. Smith MT, Neubauer DN. Cognitive behavior therapy for chronic insomnia. Clin Cornerstone 2003;5:28-40.

6. Lichstein KL, Durrence HH, Reidel BW, Bush AJ. Epidemiology of Sleep: Age, Gender and Ethnicity. Mahwah, NJ: Lawrence Erlbaum; 2004.

7. Leger D, Scheuermaier K, Philip P, Paillard M, Guilleminault C. SF-36: Evaluation of quality of life in severe and mild insomniacs compared with good sleepers. Psychosom Med 2001;63:49-55.

8. Chang PP, Ford DE, Mead LA, Cooper-Patrick L, Klag MJ. Insomnia in young men and subsequent depression: The Johns Hopkins Precursors Study. Am J Epidemiol 1997;146: 105-114.

9. Hatoum HT, Kong SX, Kania CM, Wong JM, Mendelson WB. Insomnia, health-related quality of life and healthcare resource consumption: A study of managed-care organisation enrollees. Pharmacoeconomics 1998;14:629-637.
10. Ozminkowski RJ, Wang S, Walsh JK. The direct and indirect costs of untreated insomnia in adults in the United States. Sleep 2007;30:263-273.

11. Godet-Cayre V, Pelletier-Fleury N, Le Vaillant M, Dinet J, Massuel MA, Leger D. Insomnia and absenteeism at work: Who pays the cost? Sleep 2006;29:179-184.

12. Leger D, Massuel MA, Metlaine A, SISYPHE Study Group. Professional correlates on insomnia. Sleep 2006;29:171-178.

13. Novak M, Mucsi I, Shapiro CM, Rethelyi J, Kopp MS. Increased utilization of health services by insomniacs-An epidemiological perspective. J Psychosom Res 2004;56:527536.

14. Walsh JK, Engelhardt CL. The direct economic costs of insomnia in the United States in 1995. Sleep 1999;22:S386-S393.

15. National Institutes of Health. National Institutes of Health State of the Science Conference Statement on Manifestations and Management of Chronic Insomnia in Adults, June 1315, 2005. Sleep 2005;28:1049-1057.

16. Reilly MC, Zbrozek AS, Dukes EM. The validity and reproducibility of a work productivity and activity impairment instrument. Pharmacoeconomics 1993;4:353-365.

17. Prasad M, Wahlqvist P, Shikiar R, Shih YC. A review of self-report instruments measuring health-related work productivity: A patient-reported outcomes perspective. Pharmacoeconomics 2004;22:225-0244.

18. Ware JE, Kosinski M, Dewey JE, Gandek B. How to Score and Interpret Single-Item Health Status Measures: A Manual for Users of the SF-8 Health Survey. Lincoln, RI: QualityMetric Incorporated; 2001.

19. Ware JE, Kosinski M, Keller SD. SF-36 Physical and Mental Health Summary Scales: A User's Manual. Boston, MA: The Health Institute; 1994.

20. Roth T, Roehrs T. Insomnia: Epidemiology, characteristics, and consequences. Clin Cornerstone 2003;5:5-15.

21. Machlin SR. MEPS Statistical Brief \#111: Expenses for a hospital emergency room visit, 2003. January 2006. Available at: http://meps.ahrq.gov/mepsweb/data_files/ publications/st111/stat111.pdf. Accessed January 7, 2008.

22. Machlin SR, Carper K. MEPS Statistical Brief \#149: National health care expenses in the U.S. civilian noninstitutionalized population, 2004. November 2006. Available at: http://meps .ahrq.gov/mepsweb/data_files/publications/st149/stat149 .pdf. Accessed January 7, 2008.

23. DeFrances CJ, Hall MJ. 2005 National Hospital Discharge Survey. Adv Data 2007;12:1-19.

24. Machlin SR, Carper K. MEPS Statistical Brief \#166: Expenses for office-based physician visits by specialty, 2004. March 2007. Available at: http://www.meps.ahrq.gov/mepsweb/ data_files/publications/st166/stat166.pdf. Accessed January 7, 2008

25. Best SJ, Krueger B, Hubbard C, Smith A. An assessment of the generalizability of Internet surveys. Soc Sci Com Rev 2001;19:131-145.

26. U.S. Census Bureau. Table 1128: Adult computer and adult internet users, by selected characteristics 1995 to 2006. In: Statistical Abstracts of the United States: 2008 (127 ${ }^{\text {th }}$ Edition). Washington, DC: Government Printing Office; 2008:719.

Address correspondence to: Susan C. Bolge, Ph.D.

Centocor Ortho Biotech Services 800 Ridgeview Drive Horsham, PA 19044

E-mail: sbolge@its.jnj.com 